\title{
Descripción y análisis del proceso de evaluación de la calidad de las revistas científicas españolas llevado a cabo por FECYT en el año 2008
}

\author{
M. Ángeles Coslado*, José Manuel Báez ${ }^{\star *}$, Izaskun Lacunza*
}

Resumen: La Fundación Española para la Ciencia y Tecnología (FECYT), dentro del proyecto de apoyo a la profesionalización e internacionalización de las revistas científicas españolas (ARCE), realizó en el año 2008 la primera edición de la evaluación de la calidad de las revistas científicas españolas. El objetivo principal de este estudio es doble. Por un lado, describir y analizar el proceso detallando sus características y las distintas fases de la evaluación. Por otro, establecer unas propuestas de mejora a tener en cuenta en próximas ediciones. Además, se estudian de forma cuantitativa los resultados obtenidos. A pesar de que la muestra analizada en el trabajo es relativamente pequeña, las tendencias que se observan en los resultados son de interés, puesto que reflejan de forma significativa la situación nacional, a la espera de ser confirmados al aumentar la estadística en sucesivas ediciones.

Palabras clave: Revistas científicas, evaluación, calidad, FECYT, España.

\section{Description and analysis of the process of evaluating the quality of Spanish scholarly journals, performed by FECYT in 2008}

Abstract: The Spanish Foundation for Science and Technology (FECYT), within the project to support the professionalization and internationalization of Spanish scholarly journals (ARCE), carried out the first edition of assessing the quality of Spanish scientific journals in 2008. There are two main objectives to this work. Firstly, to describe and analyze the process itself: its different features and assessment phases; secondly, to make proposals for improving future editions. In addition, we offer a quantitative study of the results. Despite the sample being relatively small, the trends observed are interesting because they reflect the national landscape and are expected to be confirmed by a greater number of statistics in future editions.

Keywords: Scholarly journals, evaluation, quality, FECYT, Spain.

* Departamento de Gestión de la Información Científica Integrada. Fundación Española para la Ciencia y la Tecnología. Correo-e: mangeles.coslado@fecyt.es.

** Exdirector del departamento de Servicios de Apoyo a la I+D+I. Fundación Española para la Ciencia y la Tecnología.

Recibido: 8-7-09; 2. ${ }^{\text {a }}$ versión 5-4-10; aceptado: 30-4-10. 


\section{Introducción}

La necesidad de publicar y difundir los resultados de las investigaciones se remonta al siglo XVII con la creación, en enero de 1665 en París, de la primera revista científica, Journal des Savants. Desde entonces y hasta nuestros días, las revistas científicas constituyen el principal medio de comunicación y conservación de los avances de la ciencia, por lo que se puede afirmar que la ciencia es "ciencia publicada" (Patalano, 2005). Las revistas científicas son aquéllas que publican, en su mayoría, artículos de investigación original, entendiendo éstos como los trabajos en los que se ha empleado una metodología científica contrastada y que aportan datos nuevos al conocimiento (Delgado López-Cózar, 2001).

A partir del siglo xx, el prestigio de las revistas desempeña un papel muy significativo en la evaluación de la producción científica y de todo el sistema científico-técnico (Patalano, 2005). Nace un gran interés en la evaluación de las revistas científicas ya que son el principal vehículo utilizado por los investigadores para dar a conocer los resultados de sus estudios (Borrego y Urbano, 2006).

En la actualidad, la publicación científica es considerada un instrumento de evaluación y constituye un factor determinante en los procesos de valoración de la actividad investigadora de individuos e instituciones (Borrego y Urbano, 2006). El prestigio de las revistas donde publican los investigadores no sólo determina el ascenso en su escala profesional, sino que también influye en la asignación de recursos económicos para la investigación (Delgado López-Cózar, 2001). Debido a esto, los responsables de la política científica están muy interesados en la valoración que se realiza a las revistas, ya que éstas son consideradas medios de expresión de una determinada comunidad científica (Ferreiro y Jiménez-Contreras, 1986).

En el caso de España, las revistas científicas que se publican son mayoritariamente de bajo impacto, por ello son los propios científicos españoles los que, a menudo, no se plantean enviar sus trabajos a publicaciones nacionales, normalmente sólo recurren a ellas cuando el artículo es de poca calidad o ha sido rechazado previamente por otra publicación extranjera de su área (si bien es cierto que esta tendencia puede tener excepciones en determinadas áreas) (Aréchaga y Fogarty, 2002). Nuestros científicos están más preocupados en publicar en revistas con factor de impacto. Hay que destacar que, según Latindex, existen 1.240 consideradas revistas de investigación científica españolas vigentes, pero un buen número son de baja calidad, con irregularidades en su producción y con escasa visibilidad exterior (Aréchaga, 2008).

En la opinión de J. M. Aréchaga, entre las debilidades que muestran las publicaciones españolas, se encuentran la deficiente profesionalización, la carencia de actividades de promoción en el exterior y el desprecio de los mejores investigadores y bibliotecarios españoles hacia las revistas científicas españolas (Aréchaga, 2008). No obstante, no todo es negativo, en España existen en la actualidad gran cantidad de científicos que podrían estar al frente de la edición de

Rev. Esp. Doc. Cient., 33, 3, julio-septiembre, 481-495, 2010. ISSN: 0210-0614. doi:10.3989/redc.2010.3.738 
revistas científicas de calidad y, con ayuda de las nuevas tecnologías y aprovechando que el nivel de la ciencia española comienza a ser nuevamente apreciado y conocido internacionalmente, es un buen momento para lograr que la ciencia publicada en revistas españolas ocupe un lugar más destacado en el panorama internacional (Aréchaga, 2008). Por tanto, en lo que hay que incidir es en la profesionalización de todos los procesos editoriales de las revistas para darles, en un primer lugar, soporte en el mercado interior y, después, en el internacional (Aréchaga, 2006).

En este contexto, la Fundación Española para la Ciencia y la Tecnología (FECYT) viene desarrollando desde el 2006 el programa "Apoyo a las revistas Científicas Españolas" (ARCE) con la principal misión de profesionalizar e internacionalizar las revistas científicas españolas. Una de las medidas adoptadas por la FECYT para mejorar la profesionalización de las revistas científicas españolas es proporcionar, de forma gratuita, una herramienta que permita la informatización del proceso editorial a las revistas que presenten una calidad contrastada.

La herramienta que se desarrolló para este cometido fue el "Repositorio Español de Ciencia y Tecnología" (RECYT). Esta aplicación informática, creada por el Centro de Supercomputación de Cataluña (CESCA), es un sistema de gestión y edición de revistas de código abierto llamado Open Journal Systems (OJS) que busca promover el acceso y así mejorar la calidad de las revistas científicas (Willinsky, 2004). La aparición de OJS está estrechamente ligada con el fenómeno Open Access (acceso abierto), que no sólo permite el acceso de forma gratuita a los artículos publicados en una revista que presenta un sistema de evaluación, sino también a los manuscritos inéditos que están disponibles en la Red.

Con el fin de darle contenido al repositorio, se invitó a participar a las revistas españolas que estaban indizadas por Thomson Reuters en alguna de las bases de datos de la Web of Knowledge, ya que presentan una calidad contrastada. Por otro lado, con el fin de seleccionar aquellas revistas que demostraran una trayectoria solvente para que formaran parte de RECYT, se llevó a cabo la I Edición de la Evaluación de la Calidad de las Revistas Científicas Españolas. De esta forma, se pretende garantizar que la ciencia española pueda mantener una voz diferenciada dentro de la Ciencia a partir de la calidad acreditada de la investigación publicada en estas revistas.

El objetivo principal de este estudio es describir y analizar el proceso de evaluación de la calidad de las revistas científicas españolas que FECYT desarrolló durante el 2008, así como exponer los resultados obtenidos en dicho proceso. Para ello, se realizará, en primer lugar, una descripción detallada de los indicadores utilizados en la evaluación, así como de la metodología llevada a cabo en cada una de las fases del proceso. Una vez descrito el sistema, se pasará a exponer los resultados y establecer unas conclusiones del análisis realizado. De este análisis, se propondrán algunas posibles mejoras a tener en cuenta en futuras ediciones. 


\section{Descripción del proceso de evaluación FECYT 2008}

El sistema de evaluación FECYT, al igual que otros sistemas de evaluación, utiliza unos indicadores basados, fundamentalmente, en tres criterios: formales, de difusión y de contenido científico (Delgado López-Cózar, 1997).

El proceso de evaluación FECYT tiene delimitado el ámbito geográfico a las revistas que se publican en España pero, en cambio, no se ha delimitado el área temática de las revistas que han sido evaluadas.

Una de las características de este modelo de evaluación es que se trata de una evaluación voluntaria y que, en el momento en el que se llevó a cabo esta primera edición, no tenía consecuencias para las agencias que evalúan la actividad científica. Así, una evaluación positiva suponía solamente el uso de la herramienta RECYT. Otra característica es que se trata de un proceso de evaluación compuesto de varias fases con ciertos indicadores de cumplimiento obligatorio para ser superadas. Finalmente, lo más novedoso, es que se trata de una evaluación contrastada, ya que se solicita a los editores de las revistas que son evaluadas una determinada documentación que justifique que efectivamente cumplen los criterios que declaran en su publicación.

\subsection{Indicadores utilizados en el proceso de evaluación}

Los indicadores utilizados en el proceso de evaluación FECYT están basados en los criterios publicados por la Fundación en el manual: La edición de revistas científicas: directrices, criterios y modelos de evaluación (Delgado López-Cózar y otros, 2006). Estos indicadores se pueden dividir en cuatro categorías relacionadas con: la calidad informativa de la revista como medio de comunicación científica, la calidad del proceso editorial, la calidad científica y la calidad de su difusión y visibilidad.

En este proceso no se establecen pesos específicos a cada uno de lo indicadores ya que, entre otras cosas, no se trabaja con puntuaciones, no se elaboran rankings y el resultado final es si supera o no el proceso.

Los indicadores evaluados que tienen que ver con la calidad informativa de la revista como medio de comunicación científica son los siguientes:

1. Identificación de los miembros de los consejos de redacción (comités editoriales) y asesores (comités científicos) de las revistas. Los miembros de ambos consejos deben estar identificados de forma clara y diferenciada, tienen que aparecer sus nombres y la filiación profesional precisa y completa o centro de trabajo para cada uno de sus miembros.

2. Si cuenta con instrucciones detalladas a los autores en relación con el envío de originales. Las instrucciones para los autores tienen que aportar información acerca de cual es la cobertura de la revista, la periodicidad y el tipo de manuscrito que admite la revista; dar recomendacio- 
nes sobre las partes que debe tener un manuscrito y sobre la correcta elaboración de títulos, resúmenes, palabras clave, firma de artículos y referencias bibliográficas. Además, tienen que indicar cual es el sistema de evaluación empleado y los criterios de publicación.

3. Si cuenta con resúmenes y palabras clave para cada uno de los artículos. Los resúmenes tienen que ser uniformes en su extensión y deben reflejar la estructura del artículo.

4. Si presenta traducción de los títulos de los artículos, de las palabras clave y de los resúmenes al inglés.

En cuanto a los indicadores relacionados con la calidad del proceso editorial, los evaluados son:

5. Declara la periodicidad y la cumple. La revista debe publicarse $\sin$ interrupción del número de fascículos que marca su periodicidad y puntualmente dentro de dicho período.

6. Si utiliza arbitraje científico en la evaluación de originales. Se considera como arbitraje científico la revisión por pares realizada por evaluadores externos.

7. Si emplea doble anonimato en la revisión externa de los trabajos, la identidad de los autores y de los revisores tiene que estar oculta.

8. Existencia de instrucciones para la revisión y si facilita a sus revisores una hoja de evaluación en la que los expertos puedan indicar los criterios y comentarios del manuscrito acerca de su originalidad, relevancia, rigor metodológico y presentación formal. Las instrucciones para los revisores aportan también información sobre cuál es el procedimiento, forma y plazos de la evaluación.

9. La comunicación de la decisión editorial adoptada sobre un trabajo y dirigida a los autores debe estar argumentada. En ella se deben incluir las razones para la aceptación, revisión o rechazo de los manuscritos, así como se tiene que facilitar a los autores los dictámenes emitidos por los evaluadores.

10. La existencia de un Consejo de Redacción. Este debe estar compuesto por el Director, Secretario de Redacción y algunos vocales. Tiene una estrecha vinculación con la organización patrocinadora de la revista y asiste a la dirección en todas sus funciones, sobre todo, en el seguimiento de los trabajos y en la definición de los contenidos y el estilo de la revista.

11. La apertura del Consejo Asesor. Al menos un tercio debe pertenecer a instituciones diferentes al organismo editor o patrocinador de la revista.

12. La apertura del Consejo de Redacción. Al menos un tercio debe pertenecer a instituciones diferentes al organismo editor o patrocinador de la revista. 
13. La internacionalidad de la revista medida a través del Consejo Asesor. Más del 20\% de los componentes del Consejo Asesor deberán ser extranjeros

Los indicadores evaluados relacionados con la calidad científica son:

14. Originalidad. Más del 50\% de los artículos deberán ser trabajos que comuniquen resultados de investigaciones que aportan nuevo conocimiento o rectifican los conocimientos anteriores.

15. La tasa de rechazo de los trabajos deberá ser igual o mayor al $40 \%$. Indica el nivel de competitividad de la revista.

16. La endogamia de los autores que publican. No se considera endogámica cuando menos del $20 \%$ de los autores pertenecen al Consejo de Redacción.

Y finalmente, los indicadores evaluados que están relacionados con la calidad de su difusión y visibilidad:

17. La revista deberá estar indizada en bases de datos internacionales y nacionales de la especialidad.

\subsection{Fases del proceso de evaluación}

El proceso de evaluación FECYT comenzó con la recopilación de datos de distintas fuentes de información: revistas indizadas en el catálogo Latindex, servicios de publicaciones de universidades y organismos públicos de investigación (OPIS), agencias y organismos de evaluación (Agencia Nacional de Evaluación de la Calidad y Acreditación (ANECA), Agencia Nacional de Evaluación y Prospectiva (ANEP) y Comisión Nacional Evaluadora de la Actividad Investigadora (CNEAI)), y catálogos de editoriales comerciales (Doyma ${ }^{1}$, Rubes Editorial y Adis). Con esta información se elaboró una lista de cerca de 1.200 revistas científicas que se publican en España, y se invitó a participar en el proceso a través del correo electrónico de la revista obtenido de las fuentes utilizadas.

Por otro lado, se realizó un formulario telemático que se distribuyó en el correo. Este formulario estaba compuesto por 56 preguntas que coincidían con los " 56 criterios FECYT para la evaluación de la calidad de las revistas españolas" (Delgado López-Cózar y otros, 2006). El objetivo del formulario, además de ser la vía para participar en la convocatoria de evaluación, es un medio por el que las revistas se autoevalúan y reflexionan sobre las prácticas que realizan. Así, a la luz del resultado obtenido del formulario cumplimentado, cada revista decide si desea ser evaluada por FECYT y de este modo comenzar la primera fase del proceso.

\footnotetext{
1 Actualmente el fondo de Doyma pertenece a Elsevier.
} 


\subsubsection{Primera fase}

Con las revistas que solicitaron la evaluación se comenzó el proceso que constó de varias fases. En la primera, se evaluaron como criterios principales y motivos de exclusión del proceso, el incumplimiento de la periodicidad y el de algunos de los requisitos mínimos a la hora de la publicación de los artículos (resúmenes, palabras clave, endogamia evidente, declaración del sistema de arbitraje que emplean, etc.).

Para evaluar los criterios de esta primera fase se solicitó a las revistas, mediante un correo electrónico, que enviaran un justificante de la empresa que imprime los números de la revista en cuestión, declarando las fechas de salida de la imprenta de los números que se le ha solicitado. Estas fechas tenían que coincidir con la periodicidad declarada por la revista.

En el caso de las revistas electrónicas se midió de forma similar a las impresas ya que las que se presentaron al proceso presentaban la declaración de la periodicidad de la forma tradicional de las publicaciones impresas. En estos casos no se les pidió que enviaran el informe de imprenta, simplemente se comprobó que los números estaban actualizados y correspondían en tiempo real a la periodicidad que declaraban.

\subsubsection{Segunda fase}

Las revistas que superaron la primera fase fueron sometidas a una segunda, en la que el sistema de arbitraje con revisores externos y la originalidad de los trabajos fueron requisitos de cumplimiento imprescindible.

Al igual que la periodicidad, la no realización de una evaluación externa de los originales se considera motivo de exclusión. En el proceso de evaluación FECYT, se considera evaluadores externos a aquellos que no pertenecen al Consejo de Redacción ni a la entidad editora de la revista y que son seleccionados ad boc para valorar la originalidad, novedad, relevancia y calidad científica de los trabajos.

Con el fin de constatar el arbitraje científico, se les solicitó a las revistas que enviaran las comunicaciones que se habían mantenido entre los agentes implicados en el proceso de revisión (autor, editor y revisor), así como los informes de evaluación de dos trabajos elegidos al azar por FECYT. Una vez comprobado el arbitraje, para juzgar si éste se apoya en una evaluación externa, se pidió también la lista de revisores (nombre y afiliaciones profesionales) que habían participado en el proceso editorial durante el 2007. Cuando el 50\% o más de los revisores pertenecían al Consejo de Redacción o a la entidad editora de la revista, se consideró que no cumplía este criterio. También se excluyeron aquellas publicaciones en las que el Consejo de Redacción tenía una implicación directa en el proceso de evaluación, por ejemplo, en los casos en los que en la evaluación participara de forma sistemática alguno de los miembros del Consejo de Redacción. Más detalles pueden ser consultados en (Coslado y otros, 2010). 


\subsubsection{Tercera fase}

La última fase del proceso consistió en presentar a las revistas que superaron las dos fases anteriores, junto con el resto de las revistas evaluadas, a una comisión de expertos formada por catedráticos de las distintas áreas del conocimiento. La comisión validó las revistas excluidas en la primera y segunda fase y evaluó, de acuerdo a su conocimiento experto, las revistas que habían superado con éxito ambas fases. La comisión, de forma consensuada y atendiendo a las características y peculiaridades de cada área temática, estableció una lista definitiva de las publicaciones que superaron la última fase del proceso de evaluación.

Se establecieron varios subcomités, según el área temática, y cada uno ponderó los indicadores apoyándose en algunos casos en otras herramientas de evaluación externa para establecer una lista definitiva que fue consensuada por todos los miembros del comité.

Para el área de Humanidades el subcomité de expertos estableció como criterio imprescindible el cumplimiento de dos de los siguientes indicadores:

- Si emplea doble anonimato en la revisión externa de los trabajos, la identidad de los autores y de los revisores tiene que estar oculta.

- La endogamia de los autores que publican. No se considera endogámica cuando menos del 20\% de los autores pertenecen al Consejo de Redacción.

- Tasa de rechazo de manuscritos superior al 20-30\%.

- La apertura del Consejo de Redacción. Al menos un tercio debe pertenecer a instituciones diferentes al organismo editor o patrocinador de la revista.

Para las áreas de: Biomedicina, Matemáticas, Física, Ingeniería y Ciencias de la Naturaleza, el subcomité estableció los siguientes criterios:

- En primer lugar, eliminaron aquellas revistas que no cumplían al menos 14 de los criterios de evaluación de FECYT.

- Las revistas que superan ese filtro se las evaluó aplicando los cuatro criterios utilizados por la Comisión de Humanidades.

- Además, tuvieron en consideración el carácter histórico de algunas revistas o pertenecer a un área de conocimiento emergente.

Para el área de Ciencias Sociales, el subcomité prestó especial interés a los siguientes indicadores:

- La tasa de rechazo de los trabajos deberá ser igual o mayor al 40\%. Indica el nivel de competitividad de la revista.

- Grado de endogamia editorial y del comité de redacción y/o el científico.

- Revistas que, aun cumpliendo criterios FECYT, al examinarlas, se comprobó que eran de divulgación aunque ésta fuera de alto nivel. 
- Cuantificación externa de su impacto (Índice de impacto de las revistas españolas de Ciencias Sociales (INRECS); Difusión y calidad de las revistas españolas (DICE), etc.

- Presencia en bases de datos. La revista deberá estar indizada en bases de datos internacionales y nacionales de la especialidad.

\section{Resultados obtenidos en la evaluación FECYT 2008}

Al cuestionario se inscribieron 275 de las cerca de 1.200 revistas que se invitaron, de las cuales 226 solicitaron la evaluación formal. A las 226 se les envió un correo electrónico solicitando la documentación necesaria para comenzar la evaluación. De estas 226 revistas, 186 enviaron la documentación solicitada y fue con éstas con las que se inició el proceso. De las 186 que fueron evaluadas, 136 superaron la primera fase por cumplir con la periodicidad y con los requisitos mínimos a la hora de la publicación de los artículos. De éstas, 87 superaron el segundo filtro de la evaluación ya que cumplieron la realización de evaluación externa por pares y la originalidad de sus trabajos. En este punto, se convocó a la comisión de expertos para que se iniciara la tercera fase para validar las dos fases anteriores y elaborar una lista definitiva de las revistas aprobadas.

De las 186 revistas evaluadas 50 fueron excluidas en la primera fase, siendo su distribución la siguiente:

- 39 no cumplían la periodicidad que declaraban.

- 8 indicaban en sus instrucciones a los autores que no tenían revisión externa o que la realizaba el Consejo de Redacción.

- 1 presentaba una endogamia evidente de los autores que publican en la revista.

- 1 no presentaba normas para los autores.

- 1 no presentaba resúmenes, ni palabras clave ni traducción al inglés.

136 revistas fueron evaluadas en la segunda fase del proceso. De ellas, 49 fueron excluidas por los siguientes motivos:

- 32 no presentaban evaluación externa, dato contrastado con la documentación solicitada.

- 6 presentaban menos de un 50\% de contenido original.

- 9 no enviaron la documentación que se les solicitó.

- 2 renunciaron al proceso.

En la tercera fase no se ha recuperado ninguna de las revistas que no habían superado las fases anteriores, por lo que la comisión validó el proceso llevado a cabo en las dos fases previas. La lista provisional de revistas aprobadas (compuesta por 87 títulos) se redujo a 33, con lo que sólo el 18\% de las revistas evaluadas inicialmente superaron el proceso de evaluación. En la figura 1 quedan reflejados los porcentajes de las revistas que han sido excluidas en cada fase.

Rev. Esp. Doc. Cient., 33, 3, julio-septiembre, 481-495, 2010. ISSN: 0210-0614. doi:10.3989/redc.2010.3.738 


\section{FIGURA 1}

Proporción de revistas que quedan excluidas en alguna de las fases del proceso de evaluación FECYT

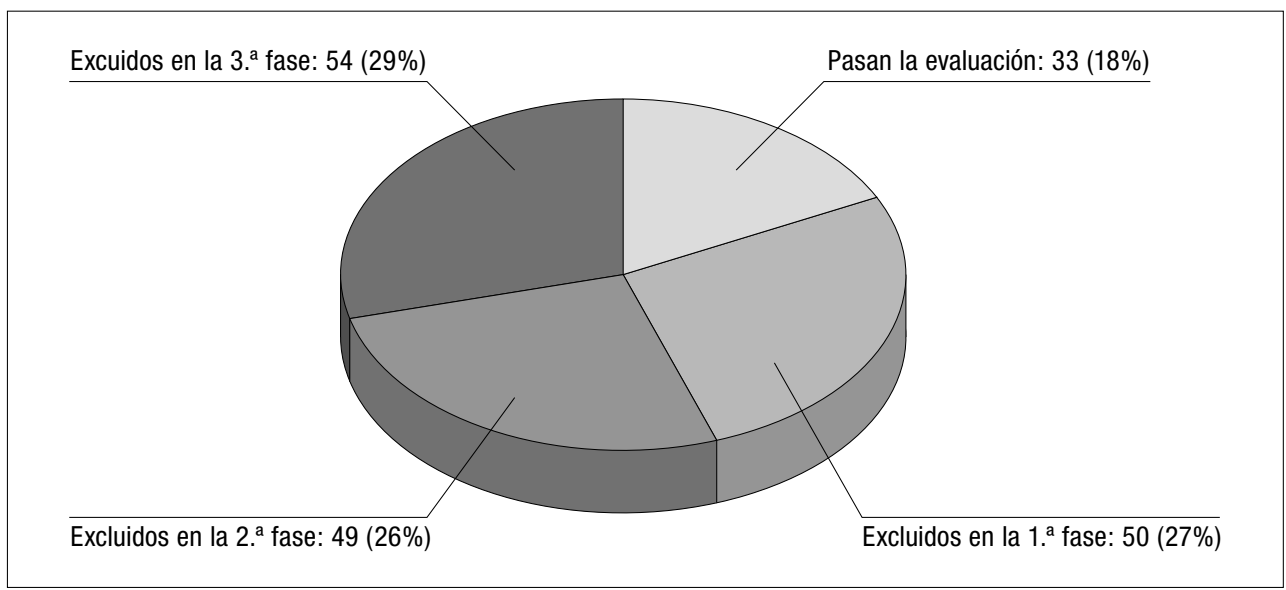

En la figura 2 se pueden observar los datos de las revistas que superaron las distintas fases del proceso de evaluación atendiendo a las distintas áreas temáticas. Cabe mencionar que algunas de las revistas fueron incluidas en varias categorías dado su carácter interdisciplinar.

\section{FIGURA 2}

Distribución por áreas temáticas de las revistas que ban superado la evaluación FECYT

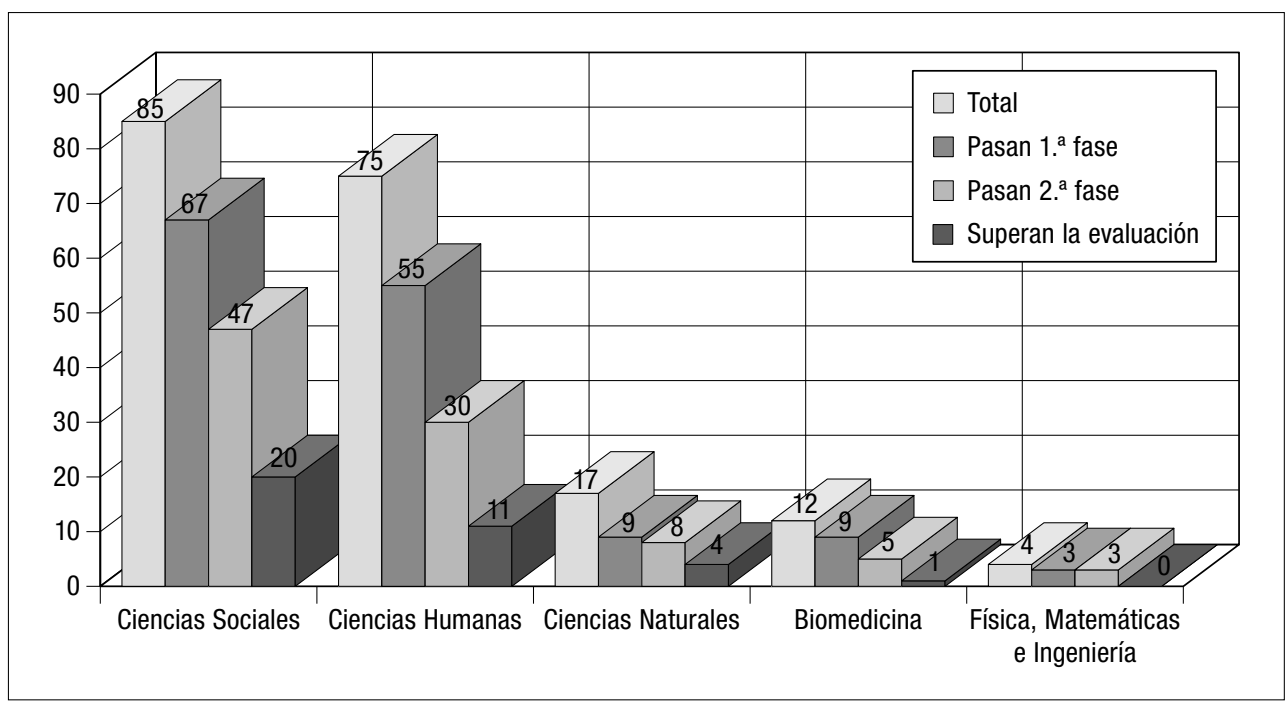


Como se puede observar en la figura 3, el mayor porcentaje de revistas que se presentaron a la evaluación pertenece al área de Ciencias Sociales, seguida de Ciencias Humanas. Por este motivo, se ha decidido establecer una división más específica en estas dos grandes áreas. Se ha establecido una subdivisión tomada de la clasificación temática de la base de datos ISOC perteneciente al Consejo Superior de Investigaciones Científicas (CSIC). En la tabla I queda reflejada la clasificación total y el porcentaje del área temática de las revistas que han superado el proceso en relación con su participación.

\section{FIGURA 3}

Distribución de las revistas según el área temática que se ban presentado a la evaluación

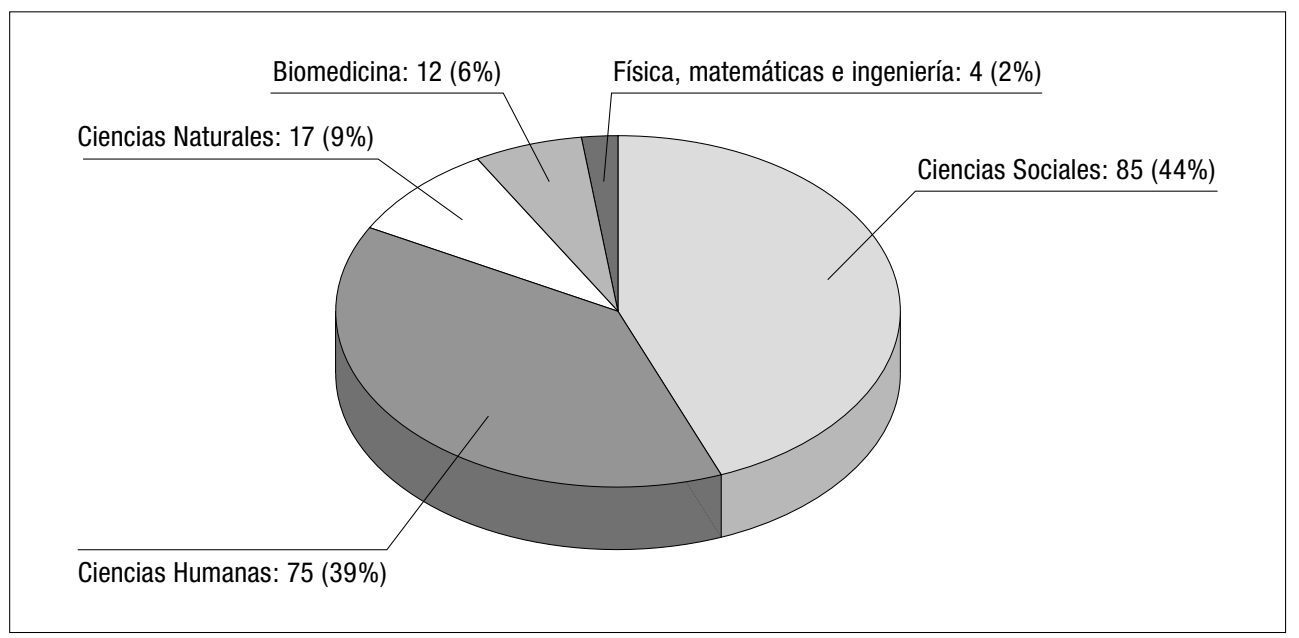

\section{Conclusiones}

El objetivo principal de este estudio es describir y analizar el proceso de evaluación de la calidad de las revistas científicas españolas llevada a cabo por la FECYT durante el año 2008. La realización de este trabajo ha servido para realizar un estudio del proceso y establecer propuestas de mejora.

A la luz de los resultados obtenidos, lo primero que hay que destacar es la baja participación de las revistas. El llamamiento se hizo a cerca de 1.200 revistas, inscribiéndose sólo 275 a la plataforma y siendo finalmente evaluadas 186 revistas que son las que enviaron la documentación solicitada. Este hecho se explica ya que, tal y como se ha expuesto en el apartado 2, la evaluación no tenía consecuencias para las agencias que evalúan la actividad científica y por tanto, la superación del proceso solamente suponía el uso de la herramienta RECYT. Hay que decir también, que RECYT no sólo alberga a las revistas que superaron la 


\section{TABLA I}

Clasificación temática de las revistas evaluadas y los porcentajes de las que ban superado la evaluación FECYT

\begin{tabular}{|c|c|c|c|}
\hline & Evaluadas & $\begin{array}{l}\text { Superan } \\
\text { el proceso }\end{array}$ & $\begin{array}{c}\% \text { de las revistas } \\
\text { que superan } \\
\text { el proceso }\end{array}$ \\
\hline Ciencias de la Educación & 20 & 6 & 30 \\
\hline Ciencias Naturales & 17 & 4 & 24 \\
\hline Ciencias Jurídicas & 17 & 4 & 24 \\
\hline Economía & 17 & 4 & 24 \\
\hline Antropología & 2 & 2 & 100 \\
\hline Historia & 15 & 2 & 13 \\
\hline Historia; Economía & 3 & 2 & 67 \\
\hline Biblioteconomía y Documentación & 6 & 1 & 17 \\
\hline Biomedicina & 12 & 1 & 8 \\
\hline Ciencias Políticas & 3 & 1 & 33 \\
\hline Geografía & 8 & 1 & 13 \\
\hline Interdisciplinar & 5 & 1 & 20 \\
\hline Lingüística & 8 & 1 & 13 \\
\hline Literatura; Lingüística & 14 & 1 & 7 \\
\hline Sociología & 9 & 1 & 11 \\
\hline Sociología; Ciencias Políticas & 1 & 1 & 100 \\
\hline América latina & 1 & 0 & 0 \\
\hline Antropología; América latina & 1 & 0 & 0 \\
\hline Arqueología-Prehistoria & 2 & 0 & 0 \\
\hline Bellas Artes & 2 & 0 & 0 \\
\hline Bellas Artes; Ciencias de la Educación & 2 & 0 & 0 \\
\hline Estudios Locales & 1 & 0 & 0 \\
\hline Filosofía & 4 & 0 & 0 \\
\hline Física, Matemáticas e Ingeniería & 4 & 0 & 0 \\
\hline Historia; América Latina & 1 & 0 & 0 \\
\hline Historia; Ciencias Jurídicas & 1 & 0 & 0 \\
\hline Interdisciplinar; Estudios Locales & 1 & 0 & 0 \\
\hline Literatura & 1 & 0 & 0 \\
\hline Literatura; América Latina & 2 & 0 & 0 \\
\hline Psicología & 6 & 0 & 0 \\
\hline Total & 186 & 33 & \\
\hline
\end{tabular}


evaluación FECYT, sino también aquéllas que han sido evaluadas por Thomson Reuters para alguna de las bases de datos de la Web of Knowledge, por lo que dichas revistas no fueron invitadas al proceso.

Estamos ante un índice de participación muy bajo por lo que se plantean las siguientes cuestiones:

- Por un lado parece que algunas revistas no concurrieran a la convocatoria por no recibir el anuncio del proceso. El llamamiento se llevó a cabo a través de un correo electrónico y por tanto, muchos sistemas pudieron haberlo dirigido a la carpeta de "correo no deseado". Además, muchos de los contactos tomados de las fuentes consultadas probablemente estaban desactualizados, por lo que no les llegó la invitación para participar en la convocatoria. Este hecho es indicativo de la baja profesionalización de las revistas ya que éstas deberían estar atentas a la información que existe de ellas en las bases de datos.

- Por otro lado, cabe pensar que el reclamo de RECYT no fue del todo suficiente y por ello muchas revistas no decidieran presentarse al proceso.

- Y por último, a la vista de los resultados obtenidos en la evaluación, el formulario pudo actuar como disuasión, ya que algunas revistas no se vieron lo suficientemente preparadas como para realizar siquiera la autoevaluación.

Una propuesta de mejora sería sin duda modificar la fórmula empleada para convocar a las revistas. Por un lado, para que la convocatoria llegue a un mayor número de publicaciones interesadas, es necesario realizar una actualización de los datos de contacto que conforman la lista de revistas de que ya se dispone. Por otro lado, la publicación en el Boletín Oficial del Estado (BOE) podría aumentar el nivel de participación.

De hecho, gracias a este análisis de la primera edición, se han podido tomar decisiones que mejoren el proceso en futuras convocatorias. En este sentido habría que decir que el 4 de noviembre de 2009 se abrió una nueva edición de la convocatoria que se publicó en el BOE el 5 de noviembre de 2009. Además, en esta nueva convocatoria ya se hace alusión a un reconocimiento de la calidad editorial y científica que otorgará la FECYT por un período de dos años. Con todo ello, se espera un aumento significativo de participación.

Por otro lado, la CNEAI, entre los criterios específicos de evaluación de los investigadores, valorará este año "los artículos publicados en revistas acreditadas por la FECYT, siempre que, a juicio del comité asesor, cuenten con una calidad científica relevante" (BOE, 1 de diciembre de 2009). Los campos en los que se aplica son: Ciencias Sociales, Políticas, del Comportamiento y de la Educación; Ciencias Económicas y Empresariales; Historia y Expresión Artística; y Filosofía, Filología y Lingüística.

Esta limitación en el número de revistas participantes no restringe el objetivo principal de este trabajo, esto es, la descripción y el análisis del proceso mismo. 
Además, puede ser útil analizar cuantitativamente la muestra resultante para obtener una visión inicial del panorama nacional ya que, aunque la muestra es reducida, las tendencias son significativas.

En este trabajo se han descrito las distintas fases que conforman el proceso de evaluación. En las dos primeras se evaluó la calidad formal, y aquellas que alcanzaron la tercera fase (el $47 \%$ del total de las revistas) fueron sometidas a una evaluación de expertos en cada área temática. Los resultados indican que, de la muestra analizada, sólo un 18\% son consideradas como revistas científicas españolas de calidad, ya que el comité de expertos ratificó la evaluación de las dos fases anteriores y seleccionó la lista de 33 revistas aprobadas. En la tercera fase se puede apreciar una cierta falta de homogeneidad de los criterios seleccionados por el comité de expertos. En las bases de la nueva convocatoria 2009 se puede observar una mayor homogeneización de los criterios a evaluar, atendiendo a las peculiaridades de las distintas áreas temáticas.

Con respecto al desequilibrio de la participación de las revistas atendiendo a la disciplina a la que pertenecen, hay que destacar que desde hace ya varios años se está llevando a cabo un gran trabajo para mejorar las revistas científicas en España, y ha sido a las revistas de Ciencias Sociales y Ciencias Humanas a las que más ha afectado. Los editores de revistas científicas pertenecientes a estas áreas están tomando conciencia de la importancia de mejorar la calidad de las revistas científicas y así mejorar la calidad científica en general.

Un motivo de peso por el que han acudido más revistas de Ciencias Sociales y Humanidades a la convocatoria FECYT, es que hay importantes instrumentos de evaluación para las revistas de estás áreas. Sin ir más lejos, la ANECA utiliza DICE (Difusión y Calidad Editorial de las Revistas Españolas de Humanidades y Ciencias Sociales y Jurídicas) como herramienta para evaluar la calidad de las publicaciones españolas en sus evaluaciones al profesorado. Esto hace que los autores se interesen en publicar en las revistas que puntúen positivamente en DICE, por lo que las revistas de estas áreas están mejorando sus técnicas y caminando hacia una mayor profesionalización e internacionalización.

Con la nueva convocatoria, en la que ya se han aplicado las mejoras propuestas y por tanto se prevé que la concurrencia de las revistas sea significativamente mayor, es de esperar que las tendencias extraídas anteriormente sean confirmadas, siendo entonces el momento idóneo para realizar un análisis cuantitativo más exhaustivo de la calidad de las revistas españolas.

\section{Agradecimientos}

Este trabajo no hubiera sido posible sin la colaboración y el asesoramiento de expertos en el área de evaluación de revistas científicas. Agradecemos el apoyo de Rafael Ruiz, Emilio Delgado y Evaristo Jiménez del grupo de investigación Evaluación de la Ciencia y de la Comunicación Científica de la Universidad de Granada; a Adelaida Román del Instituto de Estudios Documentales de Ciencia

Rev. Esp. Doc. Cient., 33, 3, julio-septiembre, 481-495, 2010. ISSN: 0210-0614. doi:10.3989/redc.2010.3.738 
y Tecnología del CSIC y a Antonio Guillamón de la UNED. También se agradecen los comentarios y sugerencias en la escritura del artículo a Germán Ros de la Universidad de Alcalá.

\section{Bibliografia}

Aréchaga, J. M. (2008). Revistas científicas españolas: donde estamos y hacia donde podríamos ir. Redvet, vol. 9 (3).

Aréchaga, J. M. (2006). Retos actuales para las revistas científicas españolas. El País, 13/09/2006.

Aréchaga, J. M., y Fogarty, David J. (2002). Publicaciones científicas profesionales en España: situación actual parámetros de calidad. Mediatika, 8, 233-245.

Borrego, A., y Urbano, C. (2006). La evaluación de revistas científicas en Ciencias Sociales y Humanidades. Información, Cultura y Sociedad, 14, 11-27.

Budapest Open Access Initiative: http://www.soros.org/openaccess/read.shtml [consultado el 1 de septiembre de 2008].

Coslado, M. A.; Lacunza, I., y Ros, G. (2010). Análisis del arbitraje científico externo en la I Edición de la Evaluación de la Calidad de Revistas Científicas Españolas realizada por FECYT en 2008 (en revisión).

Delgado López-Cózar, E.; Ruiz-Pérez, R., y Jiménez-Contreras, E. (2006). La edición de revistas científicas: directrices, criterios y modelos de evaluación, Granada: Fundación Española para la Ciencia y la Tecnología.

Delgado López-Cózar, E. (2001). Las revistas españolas de ciencias de la documentación: productos manifiestamente mejorables. El Profesional de la Información, vol. 10 (12), 46-56.

Delgado López-Cózar, E. (1997). Evaluación y aplicación de las normas de presentación de publicaciones periódicas: revisión bibliográfica. Revista Española de Documentación Científica, vol. 20 (1), 39-51.

Ferreiro, L y Jiménez-Contreras, E. (1986). Procedimientos de evaluación de las publicaciones periódicas. Estudio crítico de su empleo en las revistas científicas españolas. Revista Española de Documentación Científica, vol. 9 (1), 9-44.

Fundación Española para la Ciencia y la Tecnología: http://www.fecyt.es [consultado el 20 de marzo de 2008].

Patalano, M. (2005). Las publicaciones del campo científico: Las revistas académicas de América Latina. Anales de Documentación, vol. 8, 217-235.

Public Knowledge Project: http://pkp.sfu.ca/ [consultado el 01 de septiembre de 2008].

Repositorio Español de Ciencia y Tecnología: http://recyt.fecyt.es/ [consultado el 20 de marzo de 2008].

Sistema de Evaluación de la Calidad de las Revistas Científicas: http://evaluacionarce.fecyt. es/ [consultado el 01 de enero de 2010].

Willinsky, J. (2004). As Open Access Is Public Access, Can Journals Help Policymakers Read Research? Canadian Journal of Communication, 29, 381-401. 